\title{
TAGUNG
}

\section{Europe in Transition - Demografischer Wandel und nachhaltige Entwicklung}

\author{
Vivien Procher und Stefan Rumpf*
}

Die Europäische Union steht im Kontext der anhaltenden Globalisierung, der absehbaren demografischen Entwicklung und der zunehmenden Inanspruchnahme natürlicher Ressourcen vor der Herausforderung, eine nachhaltige Entwicklung in Europa zu ermöglichen.

Im Juni 2006 wurde daher die überarbeitete EU-Strategie zur nachhaltigen Entwicklung durch den Europäischen Rat mit dem Ziel verabschiedet, ein hohes Maß an Umweltschutz, sozialer Gerechtigkeit, sozialem Zusammenhalt und wirtschaftlichem Wohlstand zu erreichen und eine nachhaltige Entwicklung weltweit aktiv zu fördern. ${ }^{1}$ Im Mittelpunkt stehen Maßnahmen im Hinblick auf die wesentlichen Herausforderungen: Klimawandel und ,saubere' Energie, Bedrohungen für die öffentliche Gesundheit, soziale Ausgrenzung, Demografie und Migration, Bewirtschaftung natürlicher Ressourcen, nachhaltiger Verkehr sowie weltweite Armut und Entwicklung. Diese Herausforderungen sind vielfach miteinander vernetzt.

Der demografische Wandel wird Wirtschaft, Politik und Gesellschaft in der Europäischen Union in den kommenden Jahrzehnten maßgeblich beeinflussen. Analysen und Prognosen zu dessen Ausmaß und den Folgewirkungen auf Wirtschaft und Gesellschaft sind daher unabdingbare Voraussetzung für die Erarbeitung von Konzepten einer nachhaltigen Entwick-

\section{Europe in Transition - Demographic Change and Sustainable Development}

Zweite Doktoranden-Konferenz der Ruhr Graduate School in Economics (RGS Econ), in Zusammenarbeit mit dem Arbeitskreis Europäische Integration (AEI)

Gefördert durch das Jean Monnet Programm der Europäischen Kommission

Essen, 18./19. September 2008

\section{Wissenschaftliche Leitung:}

Prof. Dr. Erwin AMANN, RGS Econ; Studiendekan des Fachbereichs Wirtschaftswissenschaften, Universität Duisburg-Essen

Prof. Dr. Thomas K. BAUER, RGS Econ; RuhrUniversität Bochum

Prof. Dr. Volker CLAUSEN, RGS Econ; Universität Duisburg-Essen

Prof. Dr. Wim KÖSTERS, RGS Econ; Stellv. Vorsitzender des Vorstandes des AEI; Ruhr-Universität Bochum

Prof. Dr. Kornelius KRAFT, RGS Econ; Technische Universität Dortmund

Prof. Dr. Wolfgang LEININGER, Sprecher RGS Econ; Technische Universität Dortmund

Dr. Andreas MAURER, Schatzmeister des AEI; Stiftung Wissenschaft und Politik, Berlin

Prof. Dr. Dr. h.c. mult. Peter-Christian MÜLLER-GRAFF, Vorsitzender des Vorstandes des AEI; Ruprecht-Karls-Universität Heidelberg

Prof. Dr. Christoph M. SCHMIDT, Sprecher RGS Econ; Ruhr-Universität Bochum

Prof. Dr. Reinhold SCHNABEL, RGS Econ; Universität Duisburg-Essen

Organisatorische Leitung:

Dr. Stefan RUMPF, Koordinator RGS Econ, Essen Prof. Dr. Volker CLAUSEN

* Vivien Procher, Doktorandin, Ruhr Graduate School in Economics - RGS Econ, Essen. Dr. Stefan Rumpf, Koordinator, Ruhr Graduate School in Economics - RGS Econ; Rheinisch-Westfälisches Institut für Wirtschaftsforschung, Essen.

1 Rat der Europäischen Union: Überprüfung der EU-Strategie für nachhaltige Entwicklung - Die erneuerte Strategie, Dok. 10917/06, 26. Juni 2006. 
lung. Die europäische Integration eröffnet in diesem Prozess Optionen, die auf einzelstaatlicher Ebene nicht umsetzbar wären.

Um diese komplexen Zusammenhänge zu einem stimmigen Gesamtbild verknüpfen $\mathrm{zu}$ können, muss die wissenschaftliche Debatte auf breiter Front geführt und dabei theoriegeleitete und empirische Forschungsergebnisse zusammengeführt werden. Dies war Ziel der Veranstaltung, die sich insbesondere an Doktorandinnen und Doktoranden aus der Europäischen Union und den Beitrittsländern richtete.

\section{Nachhaltiges Wirtschaften als Herausforde-} rung für Europa

Die Konferenz wurde vom ehemaligen Exekutiv-Direktor des Umweltprogramms der Vereinten Nationen und ehemaligen Bundesumweltminister Klaus Töpfer eröffnet, der in seiner Rede ausführte, warum nachhaltiges Wirtschaften eine Chance für Europa darstellt. Nachhaltigkeit sei vor allem ein ökonomisches Prinzip. Sie werde aber fälschlicherweise häufig als rein ökologische Maxime verstanden. Nachhaltiges Handeln werde sich langfristig nur durchsetzen, wenn es sich auch wirtschaftlich lohne. Töpfer rechnet zudem damit, dass die Struktur und Leistungsfähigkeit unseres Ökosystems die nächsten Engpässe sind, mit denen es umzugehen gelte. Vor diesem Hintergrund seien viele Technologiesysteme, wie beispielsweise unsere $\mathrm{Ab}$ wassersysteme, nicht mehr zeitgemäß und auch nicht auf die ganze Welt übertragbar. Europa müsse solche Engpässe reflektieren, um Vorreiter in der Entwicklung effizienterer Technologien zu werden.

\section{Transformationsländer}

In der ersten Sitzung wurden Probleme des demografischen Wandels, der Einkommensungleichheit und andere Arbeitsmarktfragen für mittel- und osteuropäische Transformationsländer analysiert.

Gábor Antal prüfte die Robustheit des Wachstums der Einkommensungleichheit von

\section{Begrüßung}

Prof. Dr. Christoph M. SCHMIDT

Prof. Dr. Wim KÖSTERS

Prof. Dr. Ute KLAMMER, Prorektorin für Diversity Management, Universität DuisburgEssen

Prof. Dr. Erwin AMANN

Prof. Dr. Volker CLAUSEN

\section{Öffentlicher Vortrag}

Prof. Dr. Klaus TÖPFER, Exekutiv-Direktor des Umweltprogramms der Vereinten Nationen a.D.; Bundesumweltminister a.D.

\section{Transition Economies (Session 1)}

Leitung: Prof. Dr. Ansgar BELKE, RGS Econ; Universität Duisburg-Essen

Dispersion of Earned Income in Transition: The Robustness of Earnings Inequality Growth in the Hungarian Business Sector, 1986-2005

Gábor ANTAL, Central European University Budapest

The Demographic and Economic Transition in Central and Eastern Europe - Management Implications

Lasse SCHULZE, Universität Mannheim

What Hides Behind Extended Periods of Youth Unemployment? Evidence from Bosnia and Herzegovina

Nihan KOSELECI, Université Paris I - Panthéon-Sorbonne

Pensions and Savings (Session 2)

Leitung: Prof. Dr. Reinhold SCHNABEL

Retirement Saving and Attitude Towards Financial Intermediaries - Evidence for Germany

Kathrin DUMMANN, Universität Rostock

Preparing for Policy Changes: Social Security Expectations and Pension Scheme Participation Karen VAN DER WIEL, Universiteit van Tilburg

Optimal Sharing of Labor Productivity Risks and Mix of Pay-As-You-Go and Savings

Debora KUSMERSKI, Universiteit van Amsterdam

Sustainability of Public Health Systems (Session 3)

Leitung: Prof. Dr. Wolfram F. RICHTER, RGS

Econ; Technische Universität Dortmund

Ageing, Labour Supply and Consumption - Sectoral Effects of Demographic Change in Germany

Ute VOLZ, RGS Econ

Deductibles and Coinsurance Payments - A Cure for Germany?

Stefanie SCHUBERT, WHU - Otto Beisheim School of Management, Vallendar

Do Deductibles Reduce the Number of Doctor Visits? A Latent Class Panel Approach with German Data

Hendrik SCHMITZ, RGS Econ 
Vollzeitbeschäftigten in Ungarn von 1986 bis 2005. Die Streuung des realen monatlichen Einkommens sei von 1989 bis 2000 kontinuierlich angestiegen, um dann bis $2002 \mathrm{zu}$ sinken und danach wieder zu steigen.

Lasse Schulze argumentierte, dass die im Vergleich zur EU-15 zeitverzögert alternde und schrumpfende Bevölkerung eine Herausforderung für die mittel- und osteuropäischen Transformationsländer darstelle. Er zeigte vielversprechende Strategien des Personalwesens und Marketings für deren Bewältigung auf.

Nihan Koseleci untersuchte die Determinanten anhaltender Perioden der Jugendarbeitslosigkeit in Bosnien und Herzegowina mithilfe eines Mikrodatenpanels aus Haushaltsbefragungen (2001 bis 2004) auf Basis eines Duration-Modells. Die Geschwindigkeit, mit der eine junge arbeitslose Person in die Beschäftigung zurückkehrt, sei eine nicht-monotone Funktion ihrer spezifischen Charakteristika.

\section{Altersrente und Sparverhalten}

Die folgenden Beiträge befassten sich mit dem Problem der Renten und Rentenreform angesichts des demografischen Wandels. Die vorgestellten Arbeiten zeigten deutlich, dass der demografische Wandel zu Finanzierungsproblemen auf Seiten der sozialen Sicherungssysteme führt. Eine mögliche Lösung dafür stellt die Einführung einer privaten Altersvorsorge als wichtige Ergänzung zur gesetzlichen Rentenversicherung dar. Während sich die ersten beiden Forschungsarbeiten mit den Auswirkungen der aktuellen Rentenreformen auf das individuelle Sparverhalten beschäftigen, wurden im dritten Beitrag Fragen zur optimalen Bereitstellung von Sozialsicherungsleistungen im Rahmen eines theoretischen, makroökonomischen Modells behandelt.

Kathrin Dummann zeigte, dass eine erhöhte Notwendigkeit nach größeren privaten Altersrückstellungen auch $\mathrm{zu}$ einer gesteigerten Nachfrage von Finanzberatungen führt. Sie
Sustainability of Public Finances (Session 4)

Leitung: Prof. Dr. Ludger LINNEMANN, RGS

Econ; Technische Universität Dortmund

Demographic Change and Public Sector Budgets in a Federal System

Michael HOFMANN, Technische Universität Dresden

Basic Income Reform in Germany: A Microsimulation-AGE Analysis

Giulia COLOMBO, Zentrum für Europäische Wirtschaftsforschung, Mannheim

Myopic Governments and Welfare enhancing Debt Limits

Malte RIETH, RGS Econ

Preferences and Economic Policy (Session 5)

Leitung: Prof. Dr. Jeannette BROSIG, RGS

Econ; Universität Duisburg-Essen

Female Policymaker and Educational Expenditure: Cross-Country Evidence

Li-Ju CHEN, Stockholms universitet

Envy and Altruism in Children

Kirsten HÄGER, Friedrich-Schiller-Universität Jena

Inattentive Voters and Welfare State Persistence Christian BREDEMEIER, RGS Econ

Wages and Earnings (Session 6)

Leitung: Prof. Dr. Kornelius KRAFT

Estimating Union - Non-union Wage Differential in Hungary

Mariann RIGO, Central European University Budapest

International Outsourcing and the Sector Bias: New Empirical Evidence

Daniel HORGOS, Helmut-Schmidt-Universität, Hamburg

The Effect of Population Ageing on Age-Earnings and Employment Profiles in Italy

Irene MOSCA, University of Strathclyde, Glasgow

Competition and the Gender Wage Gap: New Evidence from Linked Employer-Employee Data in Hungary, 1986-2003

Anna LOVÁSZ, University of Washington; Central European University Budapest

Migration (Session 7)

Leitung: Prof. Dr. Volker CLAUSEN

Ageing and Immigration Policy in a Representative Democracy

Lena CALAHORRANO, RWTH Aachen

Integration of Immigrants in Germany: Do Enclaves Matter?

Firat YAMAN, The University of Texas at Austin

Determinants of Interregional Migration Among German States and its Implications for Reducing East-West Disparities: Results from a Panel VAR Using Efficient GMM Estimation

Timo MITZE, Ruhr-Universität Bochum; Gesellschaft für Finanz- und Regionalanalysen, Münster 
untersuchte mithilfe von deutschen Mikrodaten in einem Probit-Modell die Beratungsnachfrage sowie die Bereitschaft Finanzdienstleister zu kontaktieren. Ihre Ergebnisse zeigen, dass Personen mit hohem Einkommen und niedriger Risikobereitschaft eher den Kontakt zu einer Finanzberatung aufnehmen. Auch die Nähe zu Banken spielt dabei eine wichtige Rolle.

Karen van der Wiel untersuchte, wie subjektive Erwartungen über das gesetzliche Rentenniveau und -eintrittsalter die Teilnahme an privaten Altersvorsorgeprogrammen beeinflussen. Diese Forschungsfrage sei insbesondere für Politiker von Bedeutung, da diese wissen müssten, wie private Haushalte auf Veränderungen im sozialen Sicherungssystem reagierten. Zur Beantwortung dieser Frage verknüpfte van der Wiel Daten der holländischen Zentralbank über das Sparverhalten von Haushalten mit Daten des holländischen Pensionsbarometers. Die empirische Analyse zeigte, dass Personen, die von einer zukünftigen Kürzung der Rentenansprüche ausgingen, eher an einer privaten Altersversorgung interessiert seien. Außerdem scheine eine Herabsetzung des Renteneinstiegsalters das Sparverhalten mehr zu beeinflussen als eine Reduzierung des monatlichen Rentenniveaus. Personen mit einer niedrigen Lebenserwartung seien am meisten von einem Anstieg des Rentenalters betroffen und seien daher geneigt, mehr zu sparen. Im Allgemeinen führte die Erwartung von zukünftigen Rentenkürzungen zu einem erhöhten privaten Sparverhalten. Daraus folgten zwei wichtige politische Implikationen: Erstens, Rentenkürzungen werden zumindest teilweise durch das private Sparverhalten kompensiert. Zweitens, Politiker sollten insbesondere das Ausmaß künftiger Kürzungen deutlich kommunizieren.

Debora Kusmerski ging der Frage nach, inwiefern der Beitrag im derzeitigen Rentenumlageverfahren (,pay-as-you-go system') an die Arbeitsproduktivität gekoppelt sein sollte (Lohnindexierung). Solch eine Indexierung
Population Economics (Session 8)

Leitung: Prof. Dr. Erwin AMANN

Population Ageing and Air Pollution: The Case of Sulfur Dioxide

Jan KÜHLING, Carl von Ossietzky Universität Oldenburg

Rural Electrification and Fertility - Evidence from Côte d'Ivoire

Jörg PETERS, Rheinisch-Westfälisches Institut für Wirtschaftsforschung, Essen

Impact of Educational and Religious Homogamy on Marital Stability

Stefanie NEIMANN, RGS Econ

International Capital Mobility (Session 9)

Leitung: Prof. Dr. Jens SÜDEKUM, RGS Econ; Universität Duisburg-Essen

The Demographics of Expropriation Risk in North-South FDI

Philipp AN DE MEULEN, RWTH Aachen

FDI Location Choices: Evidence from French

First-time Movers

Vivien PROCHER, RGS Econ

habe den Vorteil, dass Risiken optimal verteilt würden. Auch habe eine Indexierung Auswirkungen auf das Verhältnis von staatlicher zu privater Altersversorgung. Würden die gesetzlichen Renten anstatt an die Löhne an das Preisniveau gekoppelt, würde dies zu einer Steigerung der privaten Altersvorsorge führen. In ihrer Arbeit benutzte Kusmerski ein überlappendes Generationenmodell und zeigte im Gegensatz zu vorherigen Forschungsarbeiten, dass gesetzliche Renten nicht ausschließlich an die Löhne gekoppelt werden sollten. Diese theoretischen Resultate stellen eine gute Begründung dar für die von vielen Ländern angestrebten Änderungen im sozialen Sicherungssystem, bei denen es nur zu einer partiellen Lohnindexierung bezüglich der Renten kommen soll.

\section{Nachhaltigkeit von öffentlichen Gesundheits- systemen}

Der demografische Wandel und seine Folgen stellen in den kommenden Jahren eine große Herausforderung für entwickelte Länder dar. Obwohl kein Wirtschaftssektor von den Folgen unberührt bleiben wird, sind es insbesondere der Gesundheits-, Bildungs- und Sozial- 
leistungssektor, die mit am meisten betroffen sein werden.

Diese Erwartungen wurden in der Arbeit von Ute Volz bestätigt. Sie quantifizierte die Effekte des demografischen Wandels in Deutschland in einem berechenbaren allgemeinen Gleichgewichtsmodell. Sie konzentrierte sich dabei auf die Veränderungen beim Arbeitsangebot und der Konsumnachfrage. Es ist der erste bekannte Beitrag, der Input-Output-Daten mit Mikrohaushaltsdaten der Sozialrechnungsmatrix verknüpft.

Die absolute und relative Ausweitung des Gesundheitssektors erhöht die Notwendigkeit, einer sektor-spezifischen Ineffizienz beim Umgang mit Ressourcen entgegenzutreten. Eine Quelle für Ineffizienz im Gesundheitswesen ist dabei das Moral Hazard Verhalten bei Krankenversicherungen. Zwei Redebeiträge haben sich mit dem Moral Hazard Verhalten beschäftigt sowie mit der Frage, ob Selbstbehalte ein angemessenes Instrument zur Kostenminderung im Gesundheitswesen darstellen.

Stefanie Schubert zeigte, dass Selbstbehalte und Teilversicherungsbeträge zwar ein mögliches, aber schwaches Instrument für die Effizienzsteigerung darstellen. Sie arbeitete mit einem berechenbaren allgemeinen Gleichgewichtsmodell und fand heraus, dass die Gesamtwirtschaft durch eine geringere Ineffizienz im Konsumverhalten schwach davon profitieren könnte. Bessere Resultate könnten erzielt werden, wenn man zu einkommensunabhängigen Gesundheitsprämien übergehen würde.

Hendrik Schmitz nutzte neue Variablen aus dem „Sozio-oekonomischen Panel“", die den individuellen Gesundheitszustand und Risikopräferenzen bezüglich der Gesundheit besser messen und es somit ermöglichten, die Preiselastizität der Nachfrage nach Gesundheitsleistungen zu bestimmen. Ein Latent-ClassAnsatz, der die Panel-Struktur der Daten ausnutzt zeige, dass der Einfluss von Selbstbe- halten auf die Anzahl der Arztbesuche vernachlässigbar sei. Eine private Zusatzversicherung dagegen erhöhe die Nachfrage nach Arztbesuchen. Demnach sei die Möglichkeit, Selbstbehalte zu wählen, kein geeignetes Mittel gegen Moral Hazard, da es eher die Selbstselektion begünstige.

Fragen zu den normativen Konsequenzen, die sich aus den wissenschaftlichen Untersuchungen ergeben, waren Hauptgegenstand der anschließenden Diskussion.

\section{Nachhaltige Finanzierung von öffentlichen Ausgaben}

Der demografische Wandel zwingt die öffentliche Hand zu Anpassungen auf der Finanzierungs- wie auf der Ausgabenseite. Die nachhaltige Finanzierbarkeit der öffentlichen Ausgabenprogramme muss daher durch geeignete politische Weichenstellungen gesichert werden. Die Beiträge dieser Sitzung widmeten sich unterschiedlichen Aspekten dieses allgemeinen Themas.

Michael Hofmann präsentierte Projektionen von nachgefragten Staatsleistungen und Beiträgen zur Finanzierung des öffentlichen Haushalts für verschiedene demografische Gruppen. Er zeigte, dass die unterschiedlichen Zuständigkeiten der Ebenen eines föderalen Staates durch die Alterung der Gesellschaft zu einem erhöhten Bedarf an Regelungen zum Finanzausgleich führen dürften, und schätzte die Wirksamkeit jüngerer politischer Reformen ab.

Giulia Colombo zeigte Simulationsergebnisse zur Wirkung einer Sozialreform mit negativer Einkommenssteuer in einem angewandten berechenbaren Gleichgewichtsmodell. Das Erfordernis, das staatliche Budget durch Anpassung der Einkommenssteuertarife auszugleichen, führte im Modell zu sehr hohen Grenzsteuersätzen auf das Arbeitseinkommen. Die Wirkungen auf das Arbeitsangebot seien insgesamt moderat, fielen aber für einzelne soziodemografische Gruppen deutlicher aus. 
Malte Rieth analysierte die aggregierten Wohlfahrtswirkungen bezogen auf die Tendenz der Regierungen zu übermäßigem Aufbau von Staatsverschuldung. Ausgehend von der Annahme, dass Regierungen wegen der Begrenztheit ihrer Amtsperioden zu kurzsichtigem Verhalten neigten und daher eine Finanzierung durch Schulden bevorzugten, zeigte er, dass eine wirksame Sanktionierung übermäßiger Verschuldung in der Art des Stabilitäts- und Wachstumspaktes die durch dieses Verhalten induzierten Wohlfahrtsverluste weitgehend reduzieren könnte.

Alle Beiträge lieferten interessante Anregungen zu den politisch notwendigen Antworten auf aktuelle Herausforderungen der öffentlichen Finanzsysteme. Zahlreiche Kommentare und Diskussionsbeiträge der Teilnehmer dieser Sitzung zielten vor allem auf Fragen der praktischen Umsetzbarkeit verschiedener Politikempfehlungen.

Individuelle Präferenzen und wirtschaftspolitische Implikationen

Die Beiträge in dieser Sitzung untersuchten, inwiefern persönliche Präferenzen wirtschaftliches Handeln bestimmen. Im Gegensatz zu den oftmals implizit vorausgesetzten Präferenzen wurden hier also ausdrücklich individuelle Präferenzen thematisiert.

Li-Ju Chen analysierte den Zusammenhang zwischen der Partizipation von Frauen in der Politik und den Aufwendungen für Bildung. Ihre länderübergreifende empirische Analyse legte nahe, dass ein höherer Anteil weiblicher Politiker mit einem höheren Anteil von Bildungsausgaben am BIP verbunden ist.

Kirsten Häger beschäftigte sich angesichts des zunehmenden Einflusses von Kindern auf ökonomisch relevante Entscheidungen mit der experimentellen Untersuchung des Verhaltens von Schulkindern verschiedener Altersklassen. Ihre Ergebnisse deuten darauf hin, dass mit steigendem Alter das Verhalten mehr von Altruismus und weniger von Neid geprägt ist.
Christian Bredemeier erklärte mit einem informationsbasierten Ansatz, dass die Einführung von Umverteilungsmaßnahmen weniger Zeit beansprucht als deren Abschaffung.

In der sich an die jeweiligen Vorträge anschließenden Diskussion wurde noch einmal die Verbindung des Vortrags zum Titel der Tagung thematisiert und dessen Beitrag zum Verständnis wirtschaftspolitischer Entscheidungen vor dem Hintergrund des demografischen Wandels deutlich gemacht.

\section{Lohn- und Einkommenseffekte}

In der folgenden Sitzung wurden Fragen der Lohnbestimmung behandelt. Hierbei wurden die Lohnfindung in den neuen und alten Mitgliedstaaten der Europäischen Union und die Auswirkungen des demografischen Wandels auf die Lohnhöhe analysiert.

Mariann Rigo präsentierte die Ergebnisse einer empirischen Studie zur Höhe des Gewerkschaftslohndifferenzials in Ungarn. Demnach sei der Effekt eher gering. Die Arbeit leistet einen wichtigen Beitrag zum Verständnis des Transformationsprozesses in Osteuropa.

Daniel Horgos betrachtete die Effekte der Auslagerung von Produktionsprozessen auf den Lohnunterschied zwischen gelernten und ungelernten Arbeitern in Deutschland. Interessanterweise führe das Outsourcing zu einem geringeren Lohnabstand, wenn es in einer Branche mit wenig Qualifizierten erfolge. Dieser Beitrag trägt zur Erklärung der Wirkung von Outsourcing als Teil der Verlagerung von Produktion in die neuen Mitgliedstaaten der Europäischen Union bei.

Irene Mosca betrachtete die Auswirkung von unterschiedlichen Kohortengrößen auf die Löhne und die Beschäftigung in Italien und verwendete ihre Ergebnisse für eine Simulation der zukünftig zu erwartenden Löhne. Dies ist ein interessanter Beitrag zu den Auswirkungen der Überalterung der Bevölkerung auf die Entlohnung. 
Anna Lovász untersuchte die Diskriminierung von Frauen. Sie überprüfte anhand von ungarischen Daten die Hypothese, dass mehr Wettbewerb $\mathrm{zu}$ weniger Diskriminierung führe. Dies ist tatsächlich der Fall. Lovász testete eine viel diskutierte Hypothese und lieferte einen Beitrag zum Verständnis der Funktionsweise von Arbeitsmärkten in Transformationsländern.

Der Wert der Arbeiten insgesamt ist darin zu sehen, dass unser Wissen über die Abläufe auf Arbeitsmärkten im vereinten Europa verbessert wird.

\section{Migration}

Die wirtschaftlichen Auswirkungen einer alternden Bevölkerung in den Industrienationen könnten teilweise auch durch eine verstärkte Zuwanderung ausländischer Arbeitskräfte gemildert werden. Im Zusammenhang mit der absehbaren demografischen Entwicklung in Deutschland, aber auch mit der EU-Osterweiterung und der diesbezüglichen Freizügigkeit von Arbeitskräften innerhalb Europas und nicht zuletzt auch im Zusammenhang mit der möglichen Entstehung von Parallelgesellschaften in den Zuwanderungsländern wie Deutschland hat dieses Thema in den letzten Jahren besondere Aufmerksamkeit erfahren. Daneben ist zu erwarten, dass die demografischen Entwicklungen bedeutsame räumliche Polarisierungseffekte nach sich ziehen, weil prosperierende Regionen mit einer relativ guten Arbeitsmarkt- und Einkommensentwicklung die jungen qualifizierten Arbeitskräfte anziehen und die abgebenden Regionen relativ zurückfallen. Die ausgewählten Beiträge untersuchten die optimale Gestaltung der Einwanderungspolitik in einer repräsentativen Demokratie, die Zusammenhänge zwischen räumlicher Konzentration von Zuwanderung und die Integration von Zuwanderern in die deutsche Gesellschaft sowie die Bestimmungsgründe der innerdeutschen Migration.

Lena Calahorrano untersuchte die Frage, wie die Alterung der Bevölkerung in Industriena- tionen deren Einwanderungspolitik beeinflusst. Ihr Ansatz verknüpfte ein ökonomisches Modell mit zwei überlappenden Generationen - mit einer jungen Generation, die arbeitet und Vermögen bildet, sowie einer älteren Generation, die nur aus den gebildeten Vermögen den Lebensunterhalt im Alter finanziert - mit einem politökonomischen Modell der parlamentarischen Demokratie. Demnach senke die Zuwanderung von Arbeitskräften unter sonst unveränderten Umständen die Reallöhne und führe zu einer Erhöhung der Kapitalrenditen. Die ältere Generation profitiere eindeutig von der höheren Entlohnung auf das gebildete Vermögen, ohne von der Lohnsenkung betroffen zu sein. Die ältere Generation werde sich daher für Zuwanderung einsetzen. Die Auswirkungen auf die jüngere Generation seien wegen der notwendigen Betrachtung über zwei Perioden etwas vielschichtiger und zum Teil gegenläufig. Trotzdem könne gezeigt werden, dass die jüngere Generation eindeutig und überwiegend aufgrund der Lohnsenkung verliere. Wenn im Zuge der Alterung der Gesellschaft das politische Gewicht der älteren Generation zunehme, werde auch das von der Politik festgelegte Ausmaß der Zuwanderung steigen. Zum Thema Nachhaltigkeit und intergenerative Gerechtigkeit könne das sehr interessante Ergebnis abgeleitet werden, dass eine auf Wiederwahl kurzfristig ausgerichtete Demokratie eine höhere Einwanderung zulassen würde, als eine Regierung mit einem langfristigen Zeithorizont, die die langfristigen Auswirkungen auf Kapitalbildung, Bevölkerungsentwicklung und Entlohnungen der Produktionsfaktoren in ihr Kalkül einbezieht.

Firat Yaman beleuchtete theoretisch wie empirisch den Zusammenhang zwischen der ethnischen räumlichen Konzentration von Einwanderern in Deutschland und dem Grad ihrer gesellschaftlichen Integration. Der Grad der Integration werde gemessen an einigen Indikatoren aus dem ,Sozio-oekonomischen Panel", wie etwa am Niveau der deutschen Sprachkenntnisse. Die daran gemessen relativ schwache Integration von Ausländern in man- 
chen Städten könnte prinzipiell auf zwei Faktoren zurückzuführen sein. Zum einen könnten in der ursprünglichen räumlichen Ansiedlungsentscheidung diejenigen Zuwanderer, die großen Wert auf kulturelle Eigenständigkeit legen, sich dort bevorzugt ansiedeln, wo bereits viele Zuwanderer mit einem ähnlichen gesellschaftlichen Hintergrund leben (Selektionseffekt). Zum anderen dürfte durch die gesellschaftliche Interaktion vor Ort in den Enklaven der Anreiz für die Zuwanderer schwächer sein, sich stärker in die deutsche Gesellschaft zu integrieren (Interaktionseffekt). Yaman kam zu dem Ergebnis, dass in der ursprünglichen räumlichen Ansiedlungsentscheidung der Zuwanderer - also im Selektionseffekt - die Hauptursache für den geringeren Grad an Integration vieler Zuwanderer zu sehen sei.

In einer sehr umfassenden ökonometrischen Studie untersuchte Timo Mitze die Determinanten der innerdeutschen Bevölkerungswanderungen zwischen den Bundesländern im Gefolge der deutschen Wiedervereinigung. Unmittelbar im Anschluss an die Wiedervereinigung sei es per Saldo zu bedeutsamen Arbeitskräftewanderungen von Ost nach West gekommen, die allerdings deutlich geringer ausfielen, als auf der Grundlage der unterschiedlichen Einkommens- und Arbeitsmarktsituation in Ost und West zu erwarten gewesen wäre. Die wahrscheinliche Ursache liege darin, dass mit den erheblichen finanziellen Transfers von West nach Ost die Erwartung einer zügigen Angleichung der Lebensverhältnisse verbunden wurde. In der zweiten Hälfte der Neunzigerjahre sei dieser Angleichungsprozess ins Stocken geraten. Aus diesem Grund sei es um die Jahrtausendwende zu einer zweiten Welle der Ost-West-Migration mit bedeutsamen Auswirkungen auf die regionale Entwicklung gekommen. Junge, gut qualifizierte Menschen seien abgewandert, wodurch das ohnehin vorhandene demografische Problem in den neuen Bundesländern noch spürbar verschärft worden sei. Eine räumlich nachhaltige Entwicklung sei damit in einigen Regionen der neuen Bundesländer kaum noch zu erreichen, es sei denn mit erheblicher politischer und finanzieller Unterstützung.

\section{Bevölkerungsökonomie}

Unter dem Gesichtspunkt der Bevölkerungsökonomie beschäftigten sich drei Beiträge mit der Frage, inwieweit gesellschaftliche Phänomene, wie Bevölkerungswachstum, Bildungsstand, technische Neuerungen oder die Alterung einer Gesellschaft, Teilaspekte einer ökologischen Nachhaltigkeit beeinflussen könnten.

Jan Kühling untersuchte in seiner Arbeit die Auswirkungen der Alterung einer Gesellschaft auf den Zusammenhang zwischen Schwefeldioxidemissionen und Bruttosozialprodukt. Er fand heraus, dass eine solche Abhängigkeit tatsächlich existiert, diese jedoch nicht monoton ist. Die Alterung der Gesellschaft führe demnach zunächst zu erhöhten Emissionen, welche bei fortschreitender Alterung hingegen zurückgingen. Eine Erklärung könne in der zunehmenden Bedeutung der Emissionen auf die Gesundheit im Alter und demnach der stärkeren Berücksichtigung in politischen Entscheidungen liegen.

Jörg Peters untersuchte die Auswirkungen der Elektrifizierung auf die Fertilität am Beispiel der Elfenbeinküste. In ländlichen Regionen sei ein signifikanter Rückgang der Fertilität zu beobachten, was am steigenden Wohlstand und der damit einhergehenden ökonomischen Sicherheit liegen könne.

Stefanie Neimann ging in ihrer Arbeit der Frage nach, inwiefern Homogamie, also die Ehe zwischen Partnern mit ähnlichen Charakteristika, Auswirkungen auf die Scheidungswahrscheinlichkeit aufweise. Trotz gegenteiliger theoretischer Annahmen lasse sich ein solcher Zusammenhang empirisch nicht nachweisen. Im Gegenteil, Partnerschaften zwischen Partnern mit geringem Bildungsstand wiesen eine signifikant höhere Scheidungswahrscheinlichkeit auf. Wesentlich für die 
Stabilität einer Partnerschaft schienen bestätigte Erwartungen der Partner zu sein.

Zwar scheinen die drei Arbeiten auf den ersten Blick sehr heterogen. Die Diskussionen zeigten jedoch, dass private Lebensumstände und gesellschaftliche Aspekte häufig untrennbar verknüpft sind und Partialanalysen diese Zusammenhänge nicht zum Ausdruck bringen können.

\section{Internationale Kapitalmobilität}

Die Beiträge zur internationalen Kapitalmobilität beschäftigten sich insbesondere mit ausländischen Direktinvestitionen und Kapitalexporten.

Der Zusammenhang zum Oberthema der Konferenz wurde hierbei insbesondere im Vortrag von Philipp an de Meulen sehr deutlich. Alternde Gesellschaften sähen sich dem Problem gegenüber, dass das erhöhte Volumen von Ersparnissen zum Zwecke der Alterssicherung zu einem Anstieg der Kapitalintensität und damit zu einem Absinken der Kapitalrenditen führe. Offene Volkswirtschaften könnten versuchen, diesem Problem dadurch zu entgehen, dass sie das inländische Kapital im arbeitsintensiven Ausland investieren, da hier höhere Kapitalrenditen zu erwarten seien. Der Beitrag von an de Meulen zeigte auf, dass dieser Politikansatz aber auch hohe Risiken berge, da viele potenzielle Empfängerstaaten aufgrund institutioneller Mängel und politischer Instabilitäten als sehr unsicher gelten müssten. Er zeigte indes auch auf, dass zumindest langfristig der Anreiz der arbeitsintensiven Empfängerstaaten zu einer Expropriation der Kapitalexporte aus den reichen Anlegerstaaten sinken könne, was letztlich zu einer erhöhten Stabilität der Rentensysteme führen würde.

Vivien Procher beschäftigte sich mit der Zielortwahl ausländischer Direktinvestitio- nen. Sie untersuchte die Determinanten dieser Entscheidung anhand französischer Erstinvestoren des Jahres 2004. Die Empfängerregionen der französischen Direktinvestitionen seien einerseits durch ein hohes Marktpotenzial gekennzeichnet. Gleichzeitig werde die Tendenz zu einem Clustering deutlich: Französische Firmen investierten gerne dort, wo bereits viele andere französische Firmen ansässig seien. Jedoch würden französische Firmen auch durch Firmencluster anderer Nationalitäten angezogen. Da Kapitalexporte und damit ausländische Direktinvestitionen in alternden Gesellschaften tendenziell zunehmen werden, analysierte der Beitrag von Procher eine wichtige Fragestellung im Rahmen des Oberthemas der Konferenz.

\section{Fazit}

Mit den Themenfeldern demografischer Wandel, nachhaltige Entwicklung und europäische Integration werden zentrale Gegenwarts- und Zukunftsthemen aufgegriffen, deren Bedeutung - aber auch Problematik - vor allem dann deutlich wird, wenn man sie, wie auf dieser Konferenz geschehen, miteinander verknüpft. Die überwiegende Zahl der vorgestellten Beiträge deckt oftmals schon zwei der drei Themenbereiche $a b$, sodass vormals getrennte Themenbereiche der Wirtschaftswissenschaften von den jungen WissenschaftlerInnen themenübergreifend analysiert werden konnten. Insbesondere die Diskussionsrunden wurden intensiv dazu genutzt, die vorgestellten Ergebnisse unter allen drei Gesichtspunkten dem demografischen Wandel, der nachhaltigen Entwicklung und der europäischen Integration - zu beleuchten. Dabei wurde deutlich, dass es noch viele offene wissenschaftliche Fragestellungen gibt, die in zukunftsweisende Forschungsgebiete münden können und noch viel Raum für weitere Konferenzen lassen. 